\title{
An Outward-Facing Aromatic Amino Acid Is Crucial for Signaling between the Membrane-Spanning and Nucleotide-Binding Domains of Multidrug Resistance Protein 1 (MRP1; ABCC1)ฐ
}

\author{
Kevin E. Weigl, Gwenaëlle Conseil, Alice J. Rothnie, ${ }^{1}$ May Arama, Yossi Tsfadia, \\ and Susan P. C. Cole \\ Department of Pathology and Molecular Medicine (K.E.W., S.P.C.C.) and Division of Cancer Biology and Genetics (G.C., A.J.R., \\ S.P.C.C.), Queen's University Cancer Research Institute, Kingston, Ontario, Canada; and Department of Biochemistry and \\ Molecular Biology, Tel Aviv University, Tel Aviv, Israel (M.A., Y.T.)
}

Received April 6, 2018; accepted June 28, 2018

\section{ABSTRACT}

The 190-kDa human MRP1 is an ATP-binding cassette multidrug and multiorganic anion efflux transporter. The 17 transmembrane helices of its three membrane-spanning domains, together with its two nucleotide binding domains (NBDs), form a stabilizing network of domain-domain interactions that ensure substrate binding in the cytoplasm is efficiently coupled to ATP binding and hydrolysis to effect solute efflux into the extracellular milieu. Here we show that Ala substitution of $\mathrm{Phe}^{583}$ in an outward-facing loop between the two halves of the transporter essentially eliminates the binding of multiple organic anions by MRP1. Conservative substitutions with Trp and Tyr had little or no effect. The F583A mutation also caused a substantial increase in orthovanadate-induced trapping of azidoADP by the cytoplasmic NBDs of MRP1, although the binding of ATP was unaffected. These observations indicate that the loss of the aromatic side chain at position 583 impairs the release of ADP and thus effectively locks the transporter in a low-affinity solute binding state. Phe ${ }^{583}$ is the first outwardfacing amino acid in MRP1 found to be critical for its transport function. Our data provide evidence for long-range coupling, presumably via allosteric interaction, between this outwardfacing region of MRP1 and both the solute binding and nucleotide binding regions of the transporter. Cryoelectron microscopy structural and homology models of MRP1 indicate that the orientation of the $\mathrm{Phe}^{583}$ side chain is altered by ATP binding but are currently unable to provide insights into the molecular mechanism by which this long-range signaling is propagated.

\section{Introduction}

Multidrug resistance protein 1 (MRP1) is a $190-\mathrm{kDa}$ integral membrane glycoprotein originally cloned from a doxorubicinselected lung cancer cell line (Cole et al., 1992; Cole, 2014). MRP1 (gene symbol $A B C C 1$ ) is found in a large number of human tumor cell lines and tumors from patients that have either acquired drug resistance through drug exposure or constitutively express this membrane transporter. Ectopic expression of MRP1 in mammalian cells confers resistance to structurally diverse

This work was supported by the Canadian Institutes of Health Research (CIHR) [Grants MOP-10519; MOP-133584] (S.P.C.C.); and the Tel Aviv University Vice-President for Research and Development fund, for Molecular Dynamics group 2017 (Y.T.). G.C. and A.J.R. were recipients of CIHR postdoctoral fellowships. S.P.C.C. was supported by the Tier 1 Canada Research Chair in Cancer Biology, and the Bracken Chair in Genetics \& Molecular Medicine.

${ }^{1}$ Current affiliation: School of Life \& Health Sciences, Aston University, Aston Triangle, Birmingham, United Kingdom.

https://doi.org/10.1124/mol.118.112615.

S This article has supplemental material available at molpharm. aspetjournals.org. natural product chemotherapeutic agents, as well as folic acid analogs [e.g., methotrexate (MTX)] and heavy metal anions including environmentally relevant arsenic metabolites (Cole et al., 1994; Leslie, 2012; Cole, 2014). MRP1 also mediates ATPdependent efflux of endogenous organic anions, many of which are glutathione (GSH), glucuronide, and sulfate conjugates. Thus, conjugated xenobiotic metabolites as well as physiologic metabolites such as the cysteinyl leukotriene $\mathrm{C}_{4}\left(\mathrm{LTC}_{4}\right)$, estradiol glucuronide $\left(\mathrm{E}_{2} 17 \beta \mathrm{G}\right)$, and estrone sulfate $\left(\mathrm{E}_{1} 3 \mathrm{SO}_{4}\right)$ are transported by MRP1 (Cole, 2014). GSH itself is also effluxed by MRP1, and several therapeutically important drugs (e.g., doxorubicin and vincristine) require the presence of this tripeptide to be exported out of cells or taken up into inside-out membrane vesicles by MRP1 (Zaman et al., 1995; Loe et al., 1996, 1998; Rappa et al., 1997; Cole, 2014). GSH (and several tripeptide analogs including $S$-methylGSH) also stimulates the binding and transport of some conjugated organic anions, such as $\mathrm{E}_{1} 3 \mathrm{SO}_{4}$, processes that are associated with significant conformational changes in MRP1 (Leslie et al., 2001; Qian et al., 2001; PeklakScott et al., 2005; Rothnie et al., 2006).

ABBREVIATIONS: ABC, ATP-binding cassette; bMrp1, bovine multidrug resistance protein 1; CFTR, cystic fibrosis transmembrane conductance regulator; cryoEM, cryoelectron microscopy; $\mathrm{E}_{1} 3 \mathrm{SO}_{4}$, estrone 3-sulfate; $\mathrm{E}_{2} 17 \beta \mathrm{G}, 17 \beta$-estradiol 17-( $\beta$-D-glucuronide); $\mathrm{ECL}$, extracellular loop; GSH, glutathione; HEK293T, human embryonic kidney cells transformed with large T antigen; ID, identification; $\mathrm{LTC}_{4}$, cysteinyl leukotriene $\mathrm{C}_{4}$; mAb, monoclonal antibody; MRP1, human multidrug resistance protein 1; MSD, membrane-spanning domain; MTX, methotrexate; NBD, nucleotide binding domain; PDB, Protein Data Bank; PXE, pseudoxanthoma elasticum; RMSD, root mean square deviation; TM, transmembrane helix. 
As a member of the "C" branch of the ATP-binding cassette $(\mathrm{ABC})$ superfamily of proteins, MRP1 contains the following five domains: three membrane-spanning domains (MSDs) with 17 transmembrane helices (TMs), and two nucleotide binding domains (NBDs) configured MSD0-MSD1-NBD1-MSD2-NBD2 (Borst et al., 2000; Keppler, 2011; Slot et al., 2011) (Fig. 1A). We and others have previously identified several discrete regions of MRP1 that are critical for the binding and/or transport of its substrates. Among these is a fragment encompassing TM10 and TM11 of MSD1 that can be photolabeled by several azido-derivatized substrates (Daoud et al., 2001; Karwatsky et al., 2003). In addition, studies employing site-directed mutagenesis have identified multiple individual amino acids within TM10 and TM11 that are essential for MRP1 activity (Koike et al., 2002, 2004; Campbell et al., 2004; Haimeur et al., 2004; Zhang et al., 2004).

A

ECL5

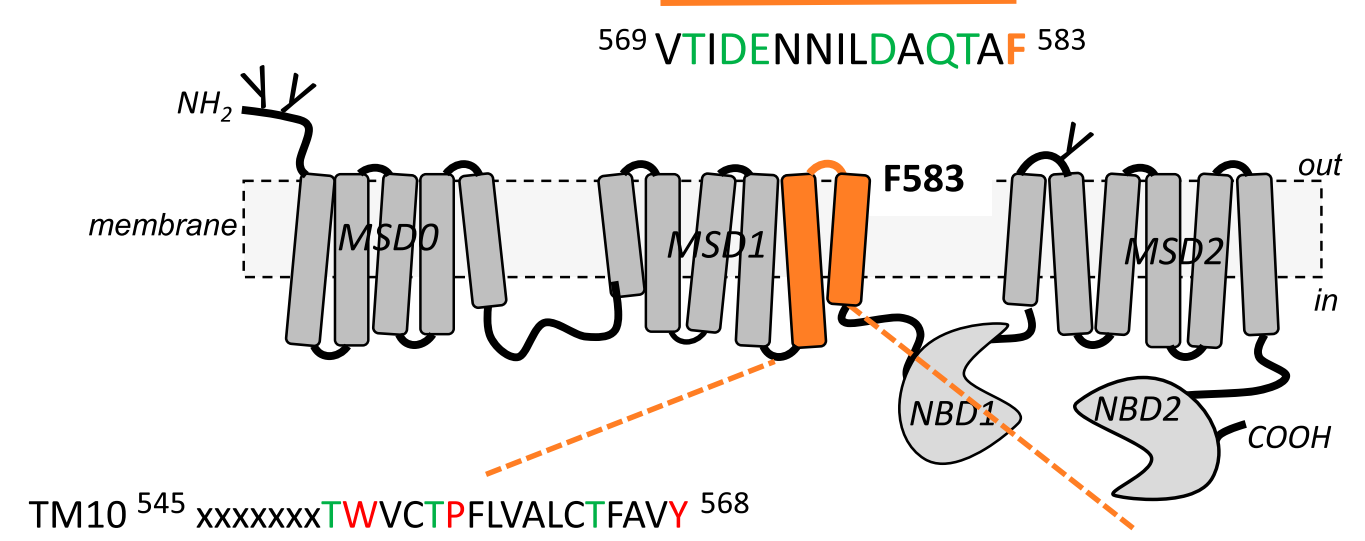

TM11 ${ }^{584}$ VSLALFNILRFPLNILPxxxxx 605

B

ECL5

552

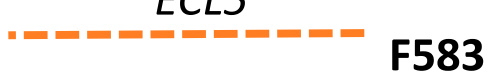

596

MRP1/ABCC1 TWVCTPFLVALCTFAVYVTIDENNILDAQTAFVSLALFNILRFPL bMrp1

CETR/ABCC 7 TWVCTPFLVALSTFAVYVTVDENN ILDAQKAFVSLALFNILRFPL

$\mathrm{ABCC} 6$

$\mathrm{MRP} 2 / \mathrm{ABCC} 2$

AFFFSGFFVVFLSVLPYALIKGI---ILRKIETTISFCIVLRMAV SFQVSTFLVALVVFAVHTLVAEN-AMNAEKAFVTLTVLNILNKAQ $\mathrm{MRP} 4 / \mathrm{ABCC} 4$ VFQLTPVLVSVVTFSVYVLVDSNN ILDAQKAFTS ITLFNILRFPL SFFSASKIIVFVTFTTYVLLGSV--ITASRVFVAVTLYGAVRLTV MRP3/ABCC3 TWMCSPFLVTLITLWVYVYVDPNNVLDAEKAFVSVSLFNILRLPL MRP 8/ABCC11 TLFIIPTVATAVWVLIHTSLKLK--LTASMA.FSMLASLNLLRLSV MRP5/ABCC5 VAPIVVVIASVVTFSVHMTLGFD--LTAAQAFTVVTVFNSMTFAL MRP7/ABCC10 LWAALPVVISIVIFITYVLMGHQ--LTATKVETALALVRMLILPL $\mathrm{ABCC} 12$ LAPIVSTIAIVLTLSCHILLRRK--LTAPVAFSVIAMFNVMKES I

SUR1/ABCC 8 MNTAIPIAAVLITEVGHVSFFKEADFSPSVA.FASLSLFHILVTPL SUR2 / ABCC 9

Ycflp MNAA I P IAAVLATEVTHA-YASGNNLKPAEA. E SLSLFHILVTPL

Yorlp

Fig. 1. Topology and sequence alignments of human MRP1 illustrating location and conservation of Phe ${ }^{583}$. (A) Topological diagram of MRP1 showing the location of Phe ${ }^{583}$ in the ECL5 between TM10 and TM11 (orange) of MSD1. Previously described mutation-sensitive amino acids in this region are indicated in red, whereas mutation-insensitive amino acids are indicated in green. (B) Sequence alignments of predicted ECL5 and flanking regions of TM10 and TM11 of human and bovine orthologs, and human and selected yeast MRP1 homologs obtained using CLUSTALW (https://npsa-prabi.ibcp.fr/ cgi-bin/npsa_automat.pl?page=npsa_clustalw.html). The alignments of TM10-ECL5-TM11 illustrate the strict (invariant) conservation of MRP1-Phe ${ }^{583}$ but varied and considerably lower conservation of the remaining ECL5 sequence in the ABCC homologs. In contrast, bovine ortholog Mrp1 is 91\% identical to human MRP1 (rat Mrp1, 87\%; mouse Mrp1, 87\%). 
According to an outward-facing atomic homology model of human MRP1 (DeGorter et al., 2008) and recent cryoelectron microscopy (cryoEM) structures of bovine Mrp1 (Johnson and Chen, 2017), the side chains of at least some non-proline mutation-sensitive amino acids in TM10 and TM11 are predicted to project into the putative substrate translocation pathway formed by MSD1 and MSD2. Thus, they may be positioned in such a way that allows direct interaction with their substrates (Amram et al., 2014). Together, these studies support the conclusion that both the geometry and the chemical properties of the amino acid side chains within TM10 and TM11 are important determinants of MRP1 substrate recognition and transport activity. In contrast, the short exofacial extracellular loop (ECL5) linking TM10 to TM11 (spanning amino acids 569-583) has never been implicated in MRP1 function (Haimeur et al., 2004; Zhang et al., 2004, 2006).

The MRP1 homologs cystic fibrosis transmembrane conductance regulator (CFTR/ABCC7) and ABCC6 (sometimes called MRP6) share approximately $20 \%$ and $45 \%$ sequence similarity with MRP1, respectively, yet have very distinct functions (Borst et al., 2000; Slot et al., 2011). Mutations in the cAMP-activated CFTR chloride channel cause cystic fibrosis, a disorder that affects mainly the lungs and the digestive system (Riordan, 2008; Hegyi et al., 2016). ABCC6 mutations, on the other hand, cause pseudoxanthoma elasticum (PXE), a disorder characterized by progressive ectopic mineralization of the skin, eyes, and cardiovascular system (LeSaux et al., 2001; Jansen et al., 2013; Uitto et al., 2014). Several disease-associated mutations have been identified in exofacial regions linking CFTR-TM5 to TM6 and ABCC6-TM10 to TM11, which corresponds to ECL5 between TM10 and TM11 of MRP1. One CFTR mutation that has been well characterized in vitro involves the phylogenetically invariant $\mathrm{Phe}^{337}$. Mutations of CFTR-Phe ${ }^{337}$ (particularly those that decrease the side chain volume of the amino acid) disrupt the anion "selectivity filter" of this chloride channel (Linsdell et al., 2000; Linsdell, 2017), a function consistent with the position of its side chain protruding into the outward-facing, water-accessible part of the channel (Corradi et al., 2015; Liu et al., 2017). A missense mutation of the analogous $\mathrm{Phe}^{568}$ of ABCC6 has been detected in patients with PXE (Le Saux et al., 2001). Thus, despite our earlier MRP1 studies indicating that ECL5 is insensitive to mutation, the aforementioned studies of CFTR and ABCC6 prompted us to investigate the functional consequences of mutating the analogous outward-facing $\mathrm{Phe}^{583}$ in MRP1 (Fig. 1).

\section{Materials and Methods}

Materials. $\left[6,7-{ }_{-}^{3} \mathrm{H}\right] \mathrm{E}_{2} 17 \beta \mathrm{G}(55 \mathrm{Ci} / \mathrm{mmol})$ and $\left[6,7-{ }^{3} \mathrm{H}\right] \mathrm{E}_{1} 3 \mathrm{SO}_{4}$ (57.3 Ci/mmol) were from PerkinElmer Life Sciences (Boston, MA), $\left[14,15,19,20-{ }^{3} \mathrm{H}\right] \mathrm{LTC}_{4}(115.3 \mathrm{Ci} / \mathrm{mmol})$ was from Amersham Biosciences (Piscataway, NJ), $\left[3^{\prime}, 5^{\prime}, 7-{ }^{3} \mathrm{H}\right] \mathrm{MTX}$ sodium salt $(17 \mathrm{Ci} / \mathrm{mmol})$ was from Moravek Inc. (Brea, CA), and 8-azido- $\left[\alpha^{-32} \mathrm{P}\right] \mathrm{ATP}(14.1 \mathrm{Ci} / \mathrm{mmol})$ was from ALT Inc. (Lexington, KY). $\mathrm{LTC}_{4}$ and MTX were from CalBiochem (San Diego, CA), and nucleotides, $\mathrm{E}_{2} 17 \beta \mathrm{G}, \mathrm{E}_{1} 3 \mathrm{SO}_{4}$, $S$-methylGSH, and sodium orthovanadate were from Sigma-Aldrich (Oakville, ON).

Generation of Mutants, Transfections, Membrane Vesicle Preparation, and Determination of MRP1 Levels. Mutations of Phe ${ }^{583}$ were introduced into pcDNA3.1(-)-MRP1 by site-directed mutagenesis, as described previously (Koike et al., 2002; Haimeur et al., 2004), with the following mutagenic primers: F583A: 5'-G GAT
GCC CAG ACA GCC GCG GTG TCT TTG GCC TTG-3'; F583W: 5'GCC CAG ACA GCC TGG GTC TCT TTG GCC TTG-3'; and F583Y: 5'-GCC CAG ACA GCC TAC GTG TCT TTG GCC TTG-3'. The fidelity of all subcloned portions of the final constructs were verified by restriction enzyme digests and sequencing. Wild-type and $\mathrm{Phe}^{583}$ mutant pcDNA3.1(-)-MRP1 expression vectors were transfected into HEK293T cells using FuGENE6 (Roche, Laval, QC, Canada) at a ratio of 1:3 (DNA/FuGENE6), as suggested by the manufacturer. Cells were collected 72 hours later, and membrane vesicles were prepared as described previously (Loe et al., 1996; Conseil et al., 2009). Proteins were quantified by the Bio-Rad Protein Assay using bovine serum albumin as standard (Bio-Rad, Hercules, CA). Levels of wild-type and mutant MRP1 were determined by immunoblot analysis of membrane vesicles prepared from transfected cells as described previously using the human MRP1-specific monoclonal antibody (mAb) QCRL-1 (Hipfner et al., 1996; Conseil et al., 2009). The binding of mAb QCRL-1 was visualized using horseradish peroxidase-conjugated goat anti-mouse IgG $(\mathrm{H}+\mathrm{L})$ (Chemicon, Temecula, CA), followed by the application of the RENAISSANCE chemiluminescence blotting substrate (PerkinElmer Life Sciences) and exposure to film. MRP1 protein levels were estimated by densitometry of the exposed film using ImageJ software (http:// rsb.info.nih.gov/ij/index.html). Equal loading of proteins was verified by amido black staining of the immunoblot membrane, as described previously (Koike et al., 2002, 2004).

MRP1-Mediated Transport of Organic Anions by Membrane Vesicles. ATP-dependent $\left[{ }^{3} \mathrm{H}\right] \mathrm{LTC}_{4}$ transport into membrane vesicles was measured using a microplate rapid filtration technique (Tabas and Dantzig, 2002; Conseil et al., 2009). Assays were performed in triplicate at $23^{\circ} \mathrm{C}$ for 1 minute in a 96 -well plate with $25-\mu l$ reactions, each containing $50 \mathrm{nM} \mathrm{LTC}_{4}(20 \mathrm{nCi} /$ reaction $), 4 \mathrm{mM}$ ATP, $10 \mathrm{mM} \mathrm{MgCl}_{2}$, an ATP regenerating system (10 mM creatine phosphate, $100 \mu \mathrm{g} / \mathrm{ml}$ creatine kinase), and $2 \mu \mathrm{g}$ of vesicle protein in transport buffer (250 mM sucrose/50 mM Tris-HCl, $\mathrm{pH}$ 7.4). Control uptake assays contained $4 \mathrm{mM}$ AMP, and the ATP regenerating system was omitted. Samples were filtered through a UniFilter-96 GF/B plate using the FilterMate Harvester (Packard BioScience, Meriden, CT), and the radioactivity was quantified using a TopCount Microplate Scintillation and Luminescence Counter (Packard). Transport in the presence of AMP was subtracted from transport in the presence of ATP to determine ATP-dependent $\left[{ }^{3} \mathrm{H}\right] \mathrm{LTC}_{4}$ uptake. Uptake of $\left[{ }^{3} \mathrm{H}\right] \mathrm{E}_{2} 17 \beta \mathrm{G}$ was measured in a fashion similar to that of components for $\left[{ }^{3} \mathrm{H}\right] \mathrm{LTC}_{4}$ transport, as described above, except that membrane vesicles $\left(4 \mu \mathrm{g}\right.$ of protein) were incubated at $37^{\circ} \mathrm{C}$ for 1 minute in a total reaction volume of $25 \mu$ l containing $400 \mathrm{nM}$ $\left[{ }^{3} \mathrm{H}\right] \mathrm{E}_{2} 17 \beta \mathrm{G}$ (40 nCi/reaction). $\left[{ }^{3} \mathrm{H}\right] \mathrm{E}_{1} 3 \mathrm{SO}_{4}$ uptake was performed at $37^{\circ} \mathrm{C}$ for 1 minute in a $25-\mu \mathrm{l}$ total reaction volume containing membrane vesicles (2.5 $\mu \mathrm{g}$ of protein), $300 \mathrm{nM}\left[{ }^{3} \mathrm{H}\right] \mathrm{E}_{1} 3 \mathrm{SO}_{4}(50 \mathrm{nCi} /$ reaction), in the presence of $3 \mathrm{mM} S$-methylGSH and the same components described above (Qian et al., 2001; Conseil et al., 2009). $\left[{ }^{3} \mathrm{H}\right] \mathrm{MTX}$ uptake was performed at $37^{\circ} \mathrm{C}$ for 20 minutes in a $50-\mu$ l total reaction volume containing membrane vesicles (2.5 $\mu \mathrm{g}$ of protein), $100 \mu \mathrm{M}$ $\left[{ }^{3} \mathrm{H}\right] \mathrm{MTX}(250 \mathrm{nCi} /$ reaction) and the same components as described above (Maeno et al., 2009).

$K_{\mathrm{m} \text { (apparent) }}$ and $V_{\max }$ values of ATP-dependent $\mathrm{LTC}_{4}$ transport by membrane vesicles ( $3 \mu \mathrm{g}$ of protein) were determined by measuring the initial rates of uptake at eight different $\left[{ }^{3} \mathrm{H}\right] \mathrm{LTC}_{4}$ concentrations (10-1000 nM) for 1 minute at $23^{\circ} \mathrm{C}$ as described above. Data were analyzed using Prism software (GraphPad Software Inc., San Diego, $\mathrm{CA}$ ), and kinetic parameters were determined by nonlinear regression and Michaelis-Menten analyses.

Photolabeling of MRP1 with $\left[{ }^{\mathbf{3}} \mathbf{H}\right] \mathbf{L T C}_{4}$. Wild-type and mutant MRP1-enriched vesicle proteins (50 $\mu \mathrm{g}$ in $30 \mu \mathrm{l})$ were labeled with the intrinsically photoactive $\left[{ }^{3} \mathrm{H}\right] \mathrm{LTC}_{4}(0.08 \mu \mathrm{Ci}, 200 \mathrm{nM})$ in the presence of $10 \mathrm{mM} \mathrm{MgCl}_{2}$. Proteins were then resolved by SDS-PAGE and processed for fluorography as described previously (Koike et al., 2004; Conseil et al., 2009). After exposing the dried gel to film at $-70^{\circ} \mathrm{C}$, the relative levels of $\left[{ }^{3} \mathrm{H}\right] \mathrm{LTC}_{4}$ photolabeling were estimated by densitometry, as was done previously. A companion immunoblot of the 
same samples probed with mAb QCRL-1 was carried out as a control to assess relative levels of MRP1 proteins.

$S$-MeGSH-Dependent $\left[{ }^{3} \mathrm{H}^{2} \mathbf{E}_{1} \mathbf{3 S O}_{4}\right.$ Binding to MRP1. Binding of $\left[{ }^{3} \mathrm{H}\right] \mathrm{E}_{1} 3 \mathrm{SO}_{4}$ to wild-type and F583A and F583Y mutant MRP1 proteins was carried out in triplicate using a radioligand equilibrium binding assay that we developed previously (Rothnie et al., 2006). Briefly, membrane vesicles (10 $\mu \mathrm{g}$ of protein) were allowed to equilibrate for 30 minutes at $23^{\circ} \mathrm{C}$ with $100 \mathrm{nM}\left[{ }^{3} \mathrm{H}\right] \mathrm{E}_{1} 3 \mathrm{SO}_{4}$ in the presence or absence of $3 \mathrm{mM} S$-methylGSH in a total volume of $50 \mu \mathrm{l}$ hypotonic buffer (50 mM HEPES, pH 7.4). Excess ice-cold wash buffer (20 mM Tris, $\mathrm{pH} 7.4,20 \mathrm{mM} \mathrm{MgCl}_{2}$ ) was added, and the samples were filtered through a UniFilter-96 GF/B plate, as described above. The $\left[{ }^{3} \mathrm{H}\right] \mathrm{E}_{1} 3 \mathrm{SO}_{4}$ on the filter was quantified by scintillation counting, and nonspecific binding in the absence of $S$-methylGSH was subtracted from total binding in the presence of $S$-methylGSH. $\left[{ }^{3} \mathrm{H}\right] \mathrm{E}_{1} 3 \mathrm{SO}_{4}$ binding by the $\mathrm{Phe}^{583}$ mutants was expressed as a percentage of $\left[{ }^{3} \mathrm{H}\right] \mathrm{E}_{1} 3 \mathrm{SO}_{4}$ binding by wild-type MRP1.

Photolabeling of MRP1 with 8-Azido-[ $\alpha_{-}{ }^{32}$ P]ATP and Orthovanadate-Induced Trapping of 8-Azido $\left[\alpha-{ }^{32}\right.$ P]ADP. Wildtype and F583A mutant MRP1-enriched membrane vesicle proteins $(20 \mu \mathrm{g})$ were photolabeled with $5 \mu \mathrm{M}$ 8-azido- $\left[\alpha-{ }^{32} \mathrm{P}\right] \mathrm{ATP}$ at $4{ }^{\circ} \mathrm{C}$ and after processing, the gel was exposed to film as previously described (Urbatsch et al., 1995; Koike et al., 2004; Conseil et al., 2009). To measure orthovanadate-induced trapping of 8-azido- $\left[\alpha-{ }^{32} \mathrm{P}\right] \mathrm{ADP}, \mathrm{MRP} 1$-enriched membrane vesicles $(20 \mu \mathrm{g})$ were incubated in $20 \mu \mathrm{l}$ of transport buffer with $5 \mu \mathrm{M}$ 8-azido- $\left[\alpha-{ }^{32} \mathrm{P}\right] \mathrm{ATP}$ in the presence or absence of $1 \mathrm{mM}$ freshly prepared sodium orthovanadate at $37^{\circ} \mathrm{C}$ for 15 minutes (Koike et al., 2004; Conseil et al., 2009). Membrane vesicles prepared from empty vector transfected HEK293T cells were included as negative controls. Photolabeled proteins were subjected to SDS-PAGE, and, after drying, the gel was exposed to film. A companion immunoblot probed with the mAb QCRL-1 was carried out as a control to confirm equal loading of MRP1 proteins.

Generation of Homology Models of Human MRP1. To generate homology models of human MRP1 based on the crystal structure of the heterodimeric TM287/288, sequence alignments of MRP1 with the bacterial transporter monomers were produced by MAFFT (http:// mafft.cbrc.jp/alignment/server/) using the default settings. This was achieved by first dividing MRP1 into the following two sections: MSD1-NBD1 (residues 300-871) and MSD2-NBD2 (residues 9711531). As for the Sav1866-based model of human MRP1 (DeGorter et al., 2008), MRP1-MSD0 (residues 1-299) were excluded because there is no homologous domain in prokaryotic organisms. Multiple sequence alignments of each of MSD1-NBD1 and MSD2-NBD2 with TM287 and TM288 were carried out with comparable regions of homologous ABCC transporters. MODELER software (version 9.18, https://salilab.org/modeller/) was then used to generate homology models using the Protein Data Bank (PDB) coordinates of "apo" TM287/288 [http://www.rcsb.org/pdb; PDB identification (ID): 4Q4H

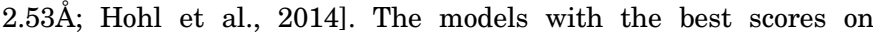
MolProbity (version 4.3; http://molprobity.biochem.duke.edu/) (Chen et al., 2010) and lower root mean square deviation (RMSD) were selected for energy minimization. They were relaxed by energy minimization using the Steepest Descent and Conjugated Gradient minimization algorithms (Petrova and Solov'ev, 1997). Each model was then validated as follows: 1) determining the RMSD between the model and the template; 2 ) evaluating the stereochemical and overall geometry using the PROCHECK (Laskowski et al., 1993) and MolProbity servers; 3) assessing Psi and Phi angles and overall secondary structure by Ramachandran plot analysis using the RAMPAGE server (http://mordred.bioc.cam.ac.uk/ rapper/rampage. php); 4) embedding the protein correctly within a 1-palmitoyl-2-oleoyl-snglycero-3-phosphocholine membrane, using both membrane prediction servers (TMHMM, TMpred) and molecular dynamics simulations using GROMACS 4.6.7 (Van Der Spoel et al., 2005) and AMBER99SB-ILDN force field (Lindorff-Larsen et al., 2010); and, finally, 5) verifying the proper equilibration of the protein in the membrane, using RMSD during the initial 100 nanoseconds of simulation, and testing the stability of the protein.

\section{Results}

Phe $^{583}$ Substitutions Alter Organic Anion Transport by MRP1. The effect of nonconservative (Ala) and conservative (Trp, Tyr) substitutions of Phe ${ }^{583}$ on MRP1 activity was determined by measuring ATP-dependent uptake of $\mathrm{LTC}_{4}$ into inside-out membrane vesicles prepared from transfected HEK293T cells. Immunoblotting of membrane vesicles with the MRP1-specific mAb QCRL-1 showed that all three mutants (F583A, F583W, and F583Y) were expressed at levels comparable to that of wild-type MRP1 (range, 60\%-140\%) (Fig. 2A). Determination of $\mathrm{LTC}_{4}$ uptake by the F583A mutant showed that it was reduced by approximately $70 \%$ relative to wild-type MRP1 (Fig. 2B) (after correcting for any differences in relative MRP1 levels; Fig. $2 \mathrm{~A}$ ). In contrast, $\mathrm{LTC}_{4}$ uptake by the conservatively substituted F583W and F583Y mutants was increased by $25 \%$ and $85 \%$, respectively.

To determine whether the mutation of $\mathrm{Phe}^{583}$ affected the transport of other organic anions by MRP1, ATP-dependent uptake of the estrogen conjugates $\mathrm{E}_{2} 17 \beta \mathrm{G}$ and $\mathrm{E}_{1} 3 \mathrm{SO}_{4}$, and the antifolate agent MTX, into inside-out membrane vesicles was measured for the three $\mathrm{Phe}^{583}$ mutants and compared
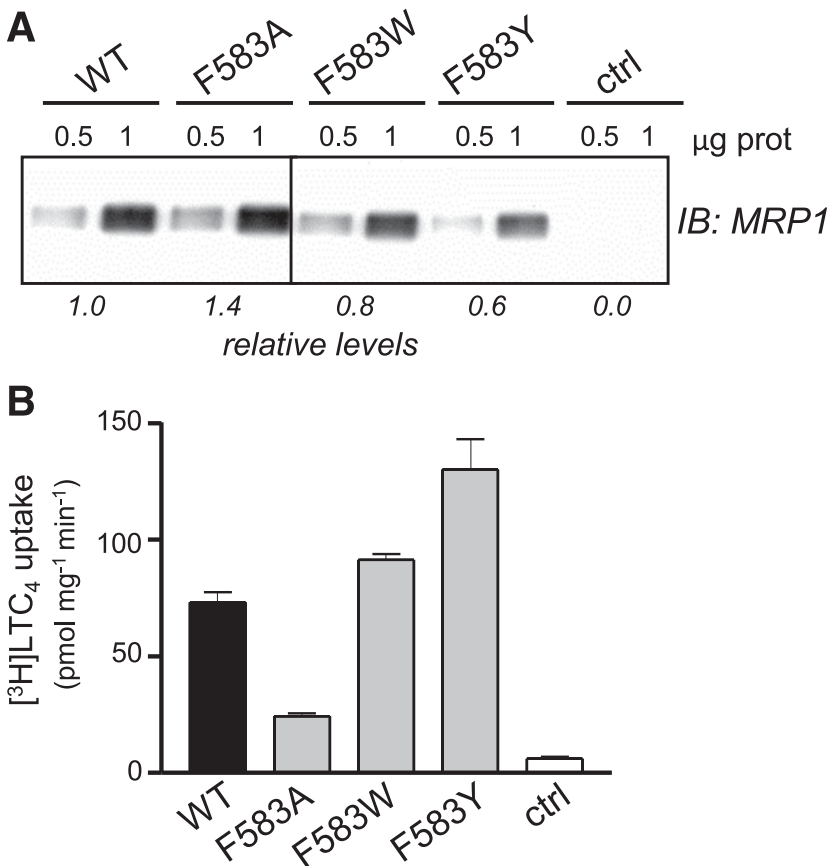

Fig. 2. Effect of $\mathrm{Phe}^{583}$ mutations on ATP-dependent transport of $\left[{ }^{3} \mathrm{H}\right] \mathrm{LTC}_{4}$ by MRP1. (A) Immunoblot (IB) of membrane vesicles prepared from HEK293T cells transfected with empty pcDNA3.1 vector (ctrl), wild-type (WT), and Phe ${ }^{583}$ mutant (F583A, F583W, and F583Y) MRP1 cDNAs. MRP1 proteins were detected with mAb QCRL-1, and the membrane was stained with amido black to confirm equal loading of proteins. The relative levels of MRP1 proteins were quantified by densitometry and are indicated under the representative immunoblot shown. The vertical lines on the image of the immunoblot indicate where extraneous lanes have been cropped out. Relative levels of MRP1 proteins in additional independent immunoblots were within $15 \%$ of those shown. (B) A representative graph of $\left[{ }^{3} \mathrm{H}\right] \mathrm{LTC}_{4}$ uptake by the membrane vesicles shown in (A). Vesicles were incubated with $\left[{ }^{3} \mathrm{H}\right] \mathrm{LTC}_{4}(50 \mathrm{nM}, 20 \mathrm{nCi})$ for 1 minute at $23^{\circ} \mathrm{C}$. All data have been corrected for differences in mutant MRP1 expression levels relative to wild-type MRP1 as needed. Each bar represents the mean $( \pm$ S.D. $)$ of triplicate determinations in a single experiment. Similar levels of $\left[{ }^{3} \mathrm{H}\right] \mathrm{LTC}_{4}$ uptake were observed in a second independent experiment and were within $10 \%$ of those shown. ctrl, membrane vesicles from cells transfected with empty pcDNA3.1 plasmid; ctrl, control; prot, protein. 

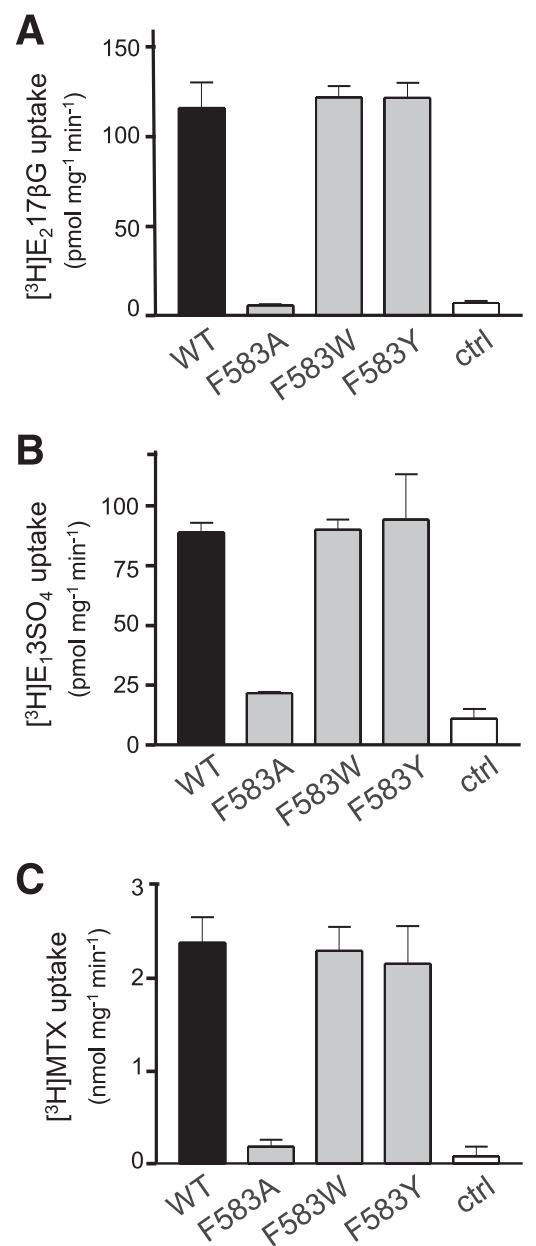

Fig. 3. Effect of $\mathrm{Phe}^{583}$ mutations on MRP1-mediated vesicular transport of estradiol glucuronide, $\mathrm{E}_{1} 3 \mathrm{SO}_{4}$, and MTX. Levels of ATP-dependent uptake of ${ }^{3} \mathrm{H}$-labeled organic anions by inside-out membrane vesicles enriched for wild-type (WT) and Phe ${ }^{583}$ mutant MRP1 proteins (F583A, F583W, and F583Y) as described in Materials and Methods: $\left[{ }^{3} \mathrm{H}\right] \mathrm{E}_{2} 17 \beta \mathrm{G}$ uptake (A); $S$-methylGSH-stimulated $\left[{ }^{3} \mathrm{H}\right] \mathrm{E}_{1} 3 \mathrm{SO}_{4}$ uptake (B); and $\left[{ }^{3} \mathrm{H}\right]$ MTX uptake (C). All data have been corrected for differences in mutant MRP1 protein levels relative to wild-type MRP1 as needed. Each bar represents the mean $( \pm$ S.D. $)$ of triplicate determinations in a single experiment. Similar levels of uptake were observed in a second independent experiment and were within $10 \%-15 \%$ of those shown. ctrl, membrane vesicles from cells transfected with empty pcDNA3.1 plasmid.

with wild-type MRP1 activity (Fig. 3). Mutation of $\mathrm{Phe}^{583}$ to Ala essentially eliminated organic anion transport by MRP1, causing a $>90 \%$ decrease in $\left[{ }^{3} \mathrm{H}\right] \mathrm{E}_{2} 17 \beta \mathrm{G}$ uptake (Fig. $3 \mathrm{~A}$ ) and a $75 \%$ decrease in $S$-methylGSH-stimulated $\left[{ }^{3} \mathrm{H}\right] \mathrm{E}_{1} 3 \mathrm{SO}_{4}$ uptake (Fig. 3B). Uptake of $\left[{ }^{3} \mathrm{H}\right] \mathrm{MTX}$ by the F583A mutant was also eliminated (Fig. 3C). In contrast, vesicular uptake of the three ${ }^{3} \mathrm{H}$-labeled organic anions by the conservatively substituted Phe ${ }^{583}$ mutants F583Y and F583W was comparable to wild-type MRP1 (Fig. 3). Taken together, these results indicate that the presence of an aromatic side chain at position 583 in MRP1 is essential for its organic anion transport activity.

Effect of Phe ${ }^{583}$ Mutations on Substrate Binding by MRP1. To determine whether the substantially reduced $\mathrm{LTC}_{4}$ transport by the F583 mutants was caused by decreased substrate binding, the intrinsically photoactive $\left[{ }^{3} \mathrm{H}\right] \mathrm{LTC}_{4}$ was used to radiolabel the wild-type and F583A mutant MRP1 proteins. As shown in Fig. 4A, a single intense 190-kDa band
A
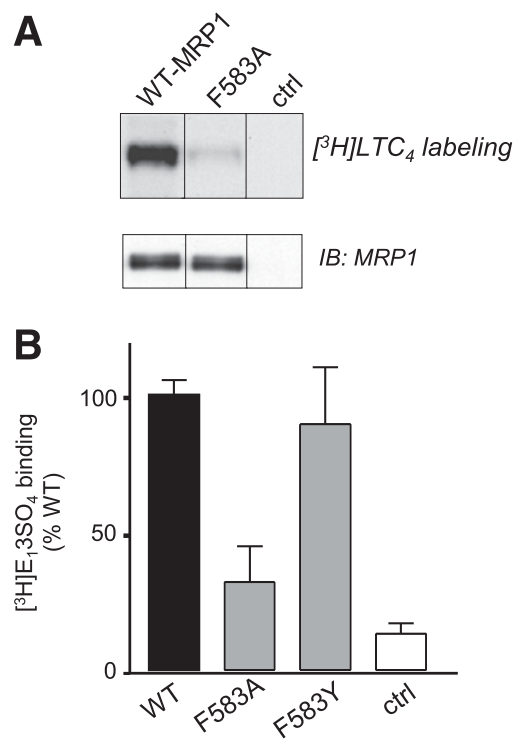

Fig. 4. Effects of $\mathrm{Phe}^{583}$ mutations on substrate binding by MRP1. (A) $\left.{ }^{3} \mathrm{H}\right] \mathrm{LTC}_{4}$ photolabeling of membrane vesicles. Top: Membrane vesicles (50 $\mu \mathrm{g}$ protein) prepared from HEK293T cells transfected with wild-type (WT) MRP1, F583A mutant, and empty pcDNA3.1 vector (ctrl) were incubated with $\left[{ }^{3} \mathrm{H}\right] \mathrm{LTC}_{4}(0.08 \mu \mathrm{Ci} ; 200 \mathrm{nM})$ and irradiated at $302 \mathrm{~nm}$, and then the radiolabeled proteins resolved by SDS-PAGE and processed for fluorography as described in Materials and Methods. Bottom: Immunoblot (IB) of membrane vesicles ( $1 \mu \mathrm{g}$ of protein) used for the photolabeling experiment. MRP1 was detected with mAb QCRL-1. Image shown is from a single blot with the vertical lines indicating where extraneous lanes have been cropped out. (B) Relative levels of $S$ methylGSH-stimulated $\left[{ }^{3} \mathrm{H}\right] \mathrm{E}_{1} 3 \mathrm{SO}_{4}$ binding to WT and $\mathrm{Phe}^{583}$ mutant F583A and F583Y MRP1 membranes. All data have been corrected for differences in Phe ${ }^{583}$ mutant (F583A, F583Y) protein levels relative to wild-type MRP1. Each bar represents the mean ( \pm S.D.) of triplicate determinations in a single experiment where levels of binding to wildtype MRP1 were $1.5 \mathrm{pmol} / \mathrm{mg}$ protein. Similar results were obtained in a second independent experiment and were within $15 \%$ of those shown.

corresponding to MRP1 was present after photolabeling membranes prepared from HEK293T cells expressing wildtype MRP1, as expected. In contrast, $\left[{ }^{3} \mathrm{H}\right] \mathrm{LTC}_{4}$-labeled MRP1 was barely detectable in F583A-enriched membrane vesicles. Thus, the decrease in $\mathrm{LTC}_{4}$ uptake by the F583A mutant appears due to a substantial loss of substrate binding.

The mechanism underlying the markedly increased $\mathrm{LTC}_{4}$ transport activity of the conservatively substituted F583Y mutant was further investigated by determining its kinetic parameters of $\left[{ }^{3} \mathrm{H}\right] \mathrm{LTC}_{4}$ uptake relative to wild-type MRP1. The $K_{\mathrm{m} \text { (apparent) }}\left(\mathrm{LTC}_{4}\right)$ for F583Y was similar to that of wildtype MRP1 (100 vs. $79 \mathrm{nM}$ ) (results not shown). On the other hand, the normalized $V_{\max }$ value of the F583Y mutant was increased $\sim 1.5$-fold relative to wild-type MRP1 (128 \pm 11 vs. $87 \pm 3 \mathrm{pmol} / \mathrm{mg}$ per minute). These results indicate that the modest increase in $\mathrm{LTC}_{4}$ transport by F583Y is associated with an increase in transport efficiency rather than any substantial alteration in uptake affinity for this organic anion.

Because the reduced $\mathrm{LTC}_{4}$ transport caused by Ala substitution of $\mathrm{Phe}^{583}$ essentially eliminated $\mathrm{LTC}_{4}$ binding (photolabeling), we wished to determine whether the binding of other substrates by this mutant was also diminished. Thus, $\left[{ }^{3} \mathrm{H}\right] \mathrm{E}_{1} 3 \mathrm{SO}_{4}$ binding (in the presence of $S$-methylGSH) to MRP1 was measured using an equilibrium binding assay (Rothnie et al., 2006). As shown in Fig. $4 \mathrm{~B},\left[{ }^{3} \mathrm{H}\right] \mathrm{E}_{1} 3 \mathrm{SO}_{4}$ binding (in the presence of $S$-methylGSH) to the F583A 
mutant was decreased by $70 \%$ relative to wild-type MRP1. In contrast, $\left[{ }^{3} \mathrm{H}\right] \mathrm{E}_{1} 3 \mathrm{SO}_{4}$ binding to the Tyr-substituted Phe ${ }^{583}$ mutant was comparable to wild-type MRP1. Thus, the differential effects of the conservative and nonconservative mutations of $\mathrm{Phe}^{583}$ on $S$-methylGSH-dependent $\mathrm{E}_{1} 3 \mathrm{SO}_{4}$ binding were similar to their effects on $S$-methylGSH-stimulated $\mathrm{E}_{1} 3 \mathrm{SO}_{4}$ transport. Since the binding assays are limited by their inability to detect specific binding of $\mathrm{E}_{1} 3 \mathrm{SO}_{4}$ alone (Rothnie et al., 2006), they cannot distinguish among the possibilities that the F583A mutation disrupts the binding of S-methylGSH, $\mathrm{E}_{1} 3 \mathrm{SO}_{4}$, or both. Nevertheless, together, these data lend support to the conclusion that the nonconservative Ala substitution of $\mathrm{Phe}^{583}$ diminishes the overall affinity of MRP1 for its organic anion substrates.

Effect of Ala Substitution of Phe ${ }^{583}$ on 8-Azido-[ $\left.\alpha-{ }^{32} P\right]$ ATP Binding and Orthovanadate-Induced Trapping of 8-Azido- $\left[\alpha-{ }^{32}\right.$ P]ADP by MRP1. Although the reduced transport activity of the nonconservatively substituted F583A MRP1 mutant appears due to reduced substrate binding, it remained possible that a common step in the transport process (i.e., interactions of the transporter with nucleotide) might also have been disrupted by this mutation. To determine whether the inactivating Ala substitution of $\mathrm{Phe}^{583}$ affected ATP binding and/or hydrolysis by MRP1, membrane vesicles enriched for wild-type MRP1 and the F583A mutant were photolabeled with 8 -azido- $\left[\alpha-{ }^{32} \mathrm{P}\right] \mathrm{ATP}$ (Conseil et al., 2009). When photolabeling was carried out at $4^{\circ} \mathrm{C}$ to minimize hydrolysis, 8-azido- $\left[\alpha-{ }^{32} \mathrm{P}\right] \mathrm{ATP}$ labeled both the wildtype and mutant MRP1 proteins with comparable intensity (Fig. 5A), indicating that the ability of the two proteins to bind ATP was similar. In contrast, when photolabeling was carried out at $37^{\circ} \mathrm{C}$ (under conditions permitting hydrolysis) and in the presence of sodium orthovanadate (Urbatsch et al., 1995), the trapping of 8 -azido- $\left[\alpha-{ }^{32} \mathrm{P}\right] \mathrm{ADP}$ by the F583A mutant was markedly increased ( $>5$-fold) relative to wild-type MRP1 (Fig. 5B). These results indicate that although loss of the aromatic side chain of $\mathrm{Phe}^{583}$ by Ala substitution does not noticeably affect nucleotide binding, the ability of MRP1 to release ADP after hydrolysis is markedly diminished, even in the absence of substrate.

Homology Models of Human MRP1. Generation of homology models of human MRP1 using the crystal structure of the outward-facing nucleotide-bound homodimeric Sav1866 exporter from Staphylococcus aureus (PDB ID: 2HYD $3 \AA$ ) as template has been previously described (Dawson and Locher, 2006; DeGorter et al., 2008). Here we generated a second human MRP1 homology model using the crystal structure of the nucleotide-free (apo) heterodimeric transporter TM287/TM288 from Thermotoga maritima (Hohl et al., 2014)

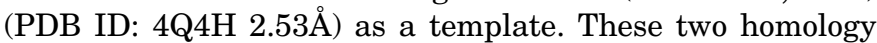
models, together with the models derived from the recent cryoEM structures of wild-type apo and $\mathrm{LTC}_{4}$-bound bovine Mrp1 (bMrp1) (Johnson and Chen, 2017), and ATP-bound, ATP hydrolysis-deficient bMrp1-E1454Q (Johnson and Chen, 2018) are shown in Figs. 6 and 7 and Supplemental Figs. 1 and 2. Comparison of the extracellular views of the two homology models (Fig. 6, A and B; Supplemental Fig. 1) shows that the orientation of the $\mathrm{Phe}^{583}$ side chain in the nucleotide-free TM287/288 model is rotated approximately $90^{\circ}$ from that in the nucleotide-bound Sav1866 model. This nucleotide binding effect on $\mathrm{Phe}^{583}$ orientation is consistent with that observed in the two nucleotide-free bMrp1 cryoEM structures
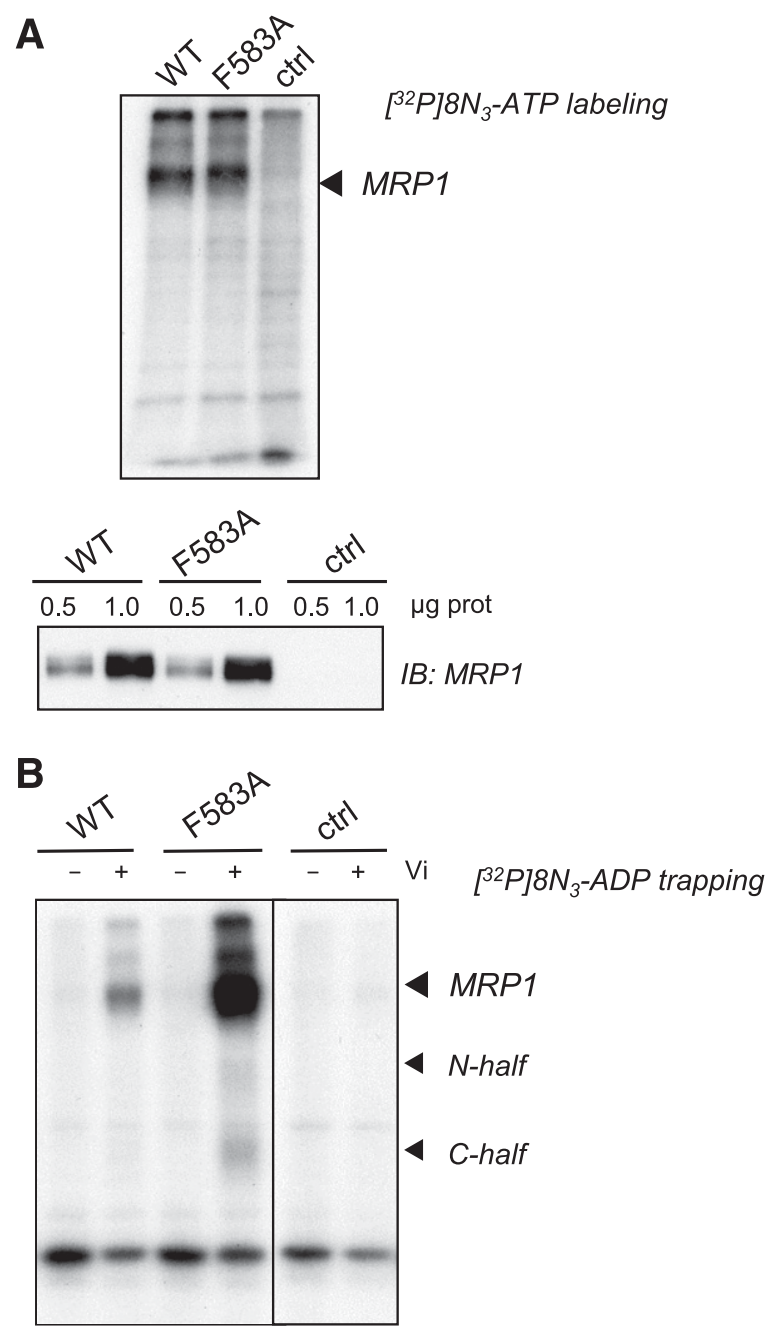

Fig. 5. Effect of Ala substitution of $\mathrm{Phe}^{583}$ on interactions of MRP1 with azido- $\left[\alpha-{ }^{32} \mathrm{P}\right]$ ATP. (A) Photolabeling of membrane vesicles from HEK293T cells transfected with wild-type (WT) MRP1, F583A mutant, and empty vector (ctrl) $\left(20 \mu \mathrm{g}\right.$ protein) with $5 \mu \mathrm{M} 8$-azido- $\left[\alpha-{ }^{32} \mathrm{P}\right] \mathrm{ATP}$ at $4^{\circ} \mathrm{C}$ as described in Materials and Methods. Shown below the film is an immunoblot (IB) of the membrane vesicles used in the photolabeling experiment. MRP1 proteins were detected with mAb QCRL-1. (B) Orthovanadate-induced trapping of 8 -azido- $\left[\alpha-{ }^{32} \mathrm{P}\right] \mathrm{ADP}$ by WT and F583A mutant MRP1. Membrane vesicles (20 $\mu \mathrm{g}$ protein) [shown in (A)] were incubated at $37^{\circ} \mathrm{C}$ with $5 \mu \mathrm{M}$ 8-azido- $\left[\alpha-{ }^{32} \mathrm{P}\right] \mathrm{ATP}$ in the absence $(-)$ or presence (+) of $1 \mathrm{mM}$ orthovanadate (Vi) for 15 minutes as described in Materials and Methods. The positions of the ${ }^{32} \mathrm{P}$-labeled $\mathrm{NH}_{2}-$ and $\mathrm{COOH}-$ proximal halves ( $N$-half and $C$-half, respectively) of MRP1 generated by mild proteolysis are indicated. The image shown is of a single autoradiogram, and the vertical line indicates where extraneous lanes have been cropped out. Results similar to those shown were observed in two additional independent experiments. prot, protein

(Fig. 6, C and D) versus the ATP-bound structure (Fig. 6E). When the side views (Supplemental Fig. 2) and cytoplasmic views (Fig. 7) are compared, additional differences among the five models are noted, particularly with respect to the NBDs. As a semiquantitative estimate of these differences, the atomic distances between the NBD1 Walker A motif and the NBD2 active transport signature motif, and between the NBD2 Walker A motif and the NBD1 active transport signature motif, of the five models were measured and are summarized in Table 1. NBD1 and NBD2 in the apo TM287/288 model of human MRP1 (Fig. 7A; Supplemental Fig. 2A) are substantially closer together (range, 8.5-16.5 ^) 
A

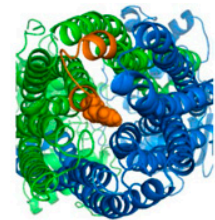

Apo TM287/288-based

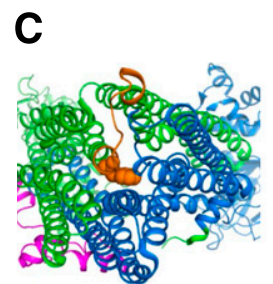

Apo bMrp1

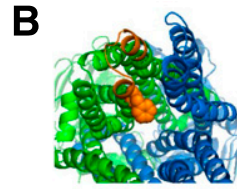

ADP + Sav1866-based

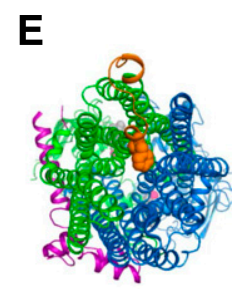

ATP + bMrp1-E1454Q
Fig. 6. Expanded extracellular view of atomic homology models of human MRP1 and cryoEM-derived models of bovine Mrp1. Shown are expanded views of the extracellular face of atomic homology models of human MRP1 derived using the crystal structure of TM287/288 (PDB ID: 4Q4H) as template (A), and Sav1866 (PDB ID: 2HYD) as template (B); and cryoEM structures of wild-type bovine Mrp1 in the absence of ATP and $\mathrm{LTC}_{4}$ (apo) (PDB ID: 5UJ9) (C) and bound to $\mathrm{LTC}_{4}$ (PDB: 5UJA) (D); and mutant, ATP hydrolysis-deficient, bovine Mrp1E1454Q bound to ATP (PDB ID: 6BHU) (E). A space-filling MRP1-Phe ${ }^{583}$ and ECL5 are shown in orange. TMs $6-11$ of MSD1 are shown in green, and TM helices 12-17 of MSD2 are blue. The sequence connecting MSD0 to MSD1 (termed the "lasso" motif) (Johnson and Chen, 2017) is shown in magenta. Full, nonexpanded extracellular views are shown in Supplemental Fig. 1. than the NBDs in either of the two apo (range, 30.5-39.4 $\AA$ ) (Fig. 7C; Supplemental Fig. 2C) or the LTC $_{4}$-bound (but nucleotide-free) (range, 20.2-25.1 Å) (Fig. 7D; Supplemental Fig. 2D) bMrp1 structures (Table 1). In contrast, the distances between the two NBDs in both the nucleotide-bound Sav1866derived model (range, 5.4-9.5 ^) (Fig. 7B; Supplemental Fig. 2B) and the ATP-bound bMrp1-E1454Q cryoEM structure (range, 5.6-8.8 ̊) (Fig. 7E; Supplemental Fig. 2E) are considerably shorter (at least 3-fold) and comparable to one another (Table 1).

\section{Discussion}

Structural studies have revealed that MSD1 and MSD2 of typical ABC transporters are composed of two bundles of six intertwined TMs with a significant twist that together form the solute translocation pathway through the membrane bilayer (Dawson and Locher, 2006; Locher, 2016). In the case of MRP1, the $\mathrm{NH}_{2}$-proximal TMs 1-5 form a third separate compact bundle (MSD0) whose interactions with the four-domain core of the transporter remain poorly defined (Rosenberg et al., 2010; Johnson and Chen, 2017). One helix bundle of MRP1 consists of TMs 6, 7, 8, 11, 15, and 16, and the other of TMs 9, 10, 12, 13, 14, and 17 (Dawson and Locher,
2006; Johnson and Chen, 2017). MRP1-ECL5 spans these two bundles when viewed from outside the cell by connecting TM10 to TM11, and thus may serve as a bridge facilitating communication between them during the solute binding and transport processes. In the present study, a nonconservative, cavity-creating (Ala) substitution of $\mathrm{Phe}^{583}$ at the $\mathrm{COOH}$ terminal end of ECL5 as it turns into TM11 was shown to cause a substantial and nonselective decrease in MRP1 transport activity (Figs. 2 and 3). This loss of activity was characterized not only by a loss of substrate binding (Fig. 4), but also by changes in the ATP interactions of the transporter (Fig. 5). Conservative substitutions of $\mathrm{Phe}^{583}$ with either a polar aromatic Tyr or Trp had no major deleterious effects on MRP1 activity, suggesting that the aromaticity of the side chain at position 583 in the ECL between TM10 and TM11 plays a dual role in maintaining both the substrate binding capacity and the transport mechanism (ATPase activity) of MRP1.

Phe ${ }^{583}$ is the first mutation-sensitive amino acid identified in the outward-facing ECL5 of MRP1. Previously, we have shown that mutations of six other amino acids $\left(\mathrm{Thr}^{570}, \mathrm{Asp}^{572}\right.$, $\mathrm{Glu}^{573}, \mathrm{Asp}^{578}, \mathrm{Gln}^{580}$, and $\mathrm{Thr}^{581}$ ) in this region have no effect on either MRP1 levels or its transport activity (Haimeur et al., 2004; Zhang et al., 2006). Although Ala substitution of Phe $\mathrm{P}^{583}$
A

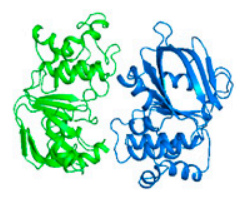

Apo TM287/288-based
B

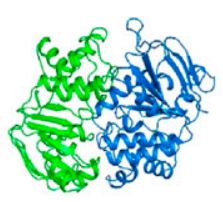

ADP + Sav1866-based
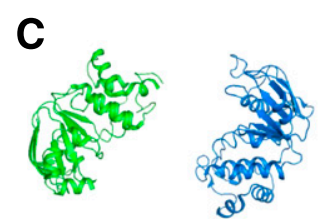

D

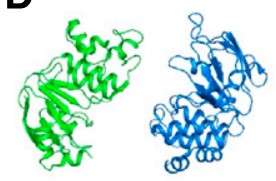

E

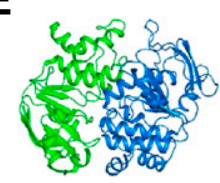

Fig. 7. Views of the NBD dimers of atomic homology models of human MRP1 and cryoEM-derived models of bovine Mrp1. Shown are views from the cytoplasm along the membrane normal of the NBD dimers from atomic homology models of human MRP1 derived using the crystal structure of TM287/288 (PDB ID: 4Q4H) as template (A) and Sav1866 (PDB ID: 2HYD) as template (B); cryoEM structures of wild-type bovine Mrp1 in the absence of ATP and $\mathrm{LTC}_{4}$ (apo) (PDB ID: 5UJ9) (C) and bound to $\mathrm{LTC}_{4}$ (PDB ID: 5UJA) (D); and mutant, ATPhydrolysis deficient, bovine Mrp1-E1454Q bound to ATP (PDB ID: $6 \mathrm{BHU}$ ) (ATP and $\mathrm{Mg}^{2+}$ have been removed for clarity) (E). NBD1 connected to TM11/MSD1 is shown in green, and NBD2 connected to TM17/MSD2 is in blue. 
TABLE 1

Interatomic distances between NBD1 and NBD2 of MRP1/bMrp1 homology and cryoEM models

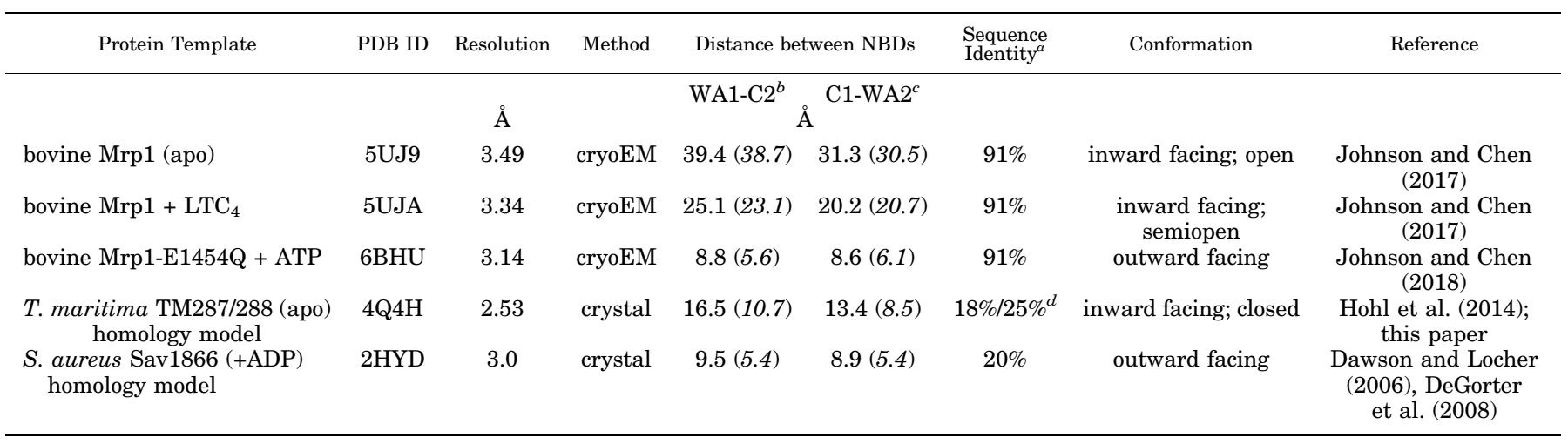

${ }^{a}$ Sequence identity with human MRP1.

${ }^{b}$ Distances between NBDs represented by distance between N $\zeta$ atom of Lys (or, in italics and in parentheses, C $\alpha$ of Gly) in NBD1-W $\mathrm{A}_{\mathrm{A}}$ motif and C $\alpha$ of Ser in NBD2 active transport signature motif.

${ }^{c}$ Distance between NBDs represented by distance between $\mathrm{C} \alpha$ of Ser in NBD1 active transport signature motif and N $\zeta$ atom of Lys (or, in italics and parentheses, C $\alpha$ of Gly) in $\mathrm{NBD} 2-\mathrm{W}_{\mathrm{A}}$ motif.

${ }^{d} 18 \%$ identity when aligned with MRP1 MSD1 + NBD1; 25\% identity when aligned with MRP1 MSD2 + NBD2.

essentially abrogates $\mathrm{LTC}_{4}$ and $S$-methylGSH-dependent $\mathrm{E}_{1} 3 \mathrm{SO}_{4}$ binding by MRP1, its outward-facing position precludes the direct involvement of this amino acid in initial substrate binding. Rather, our data suggest that the presence of an aromatic side chain at position 583 is indirectly critical for maintaining the integrity of an MRP1 binding pocket that is accessible to its organic anion substrates from the cytoplasm. In earlier studies, we demonstrated that Ala substitution (but not Trp or Phe substitution) of the more distal polar aromatic Tyr ${ }^{568}$ (at the TM10/ECL5 interface) also altered MRP1 function, but only in a very selective and relatively moderate way (i.e., $\mathrm{E}_{2} 17 \beta \mathrm{G}$ transport was decreased but $\mathrm{LTC}_{4}$ transport and drug resistance were unaffected) (Zhang et al., 2006). Thus, the phenotypes of the Y568A and F583A mutants are quite dissimilar, despite the location of $\mathrm{Tyr}^{568}$ and $\mathrm{Phe}^{583}$ in the same exofacial region of MRP1. These observations indicate that the mechanistic roles of these two mutation-sensitive aromatic amino acids in substrate binding and transport by MRP1 are quite distinct.

To our knowledge, PXE-associated mutations of ABCC6$\mathrm{Phe}^{568}$ (analogous to MRP1-Phe ${ }^{583}$ ) have not been phenotypically characterized. However, mutations of the analogous CFTR-Phe ${ }^{337}$ have been considered extensively, with the most relevant to the current study being CFTR-F337A, which alters the anion selectivity of this chloride channel (Linsdell, 2017). In contrast to the selective effects of CFTR-F337A, we observed the MRP1-F583A mutant to be profoundly defective in the binding and transport of multiple organic anions. This indicates that Ala substitution of $\mathrm{Phe}^{583}$ is much more disruptive to MRP1 function than its counterpart is to CFTR function. Ala substitution of the analogous Phe in two Saccharomyces cerevisiae MRP-related Abcc proteins (i.e., $\mathrm{Phe}^{565}$ in the five-domain vacuolar Ycf1p and $\mathrm{Phe}^{568}$ in the four-domain plasma membrane Yor1p) did not adversely affect the cadmium and oligomycin resistance-conferring properties, respectively, of these transporters (Wei et al., 2016). Thus, the essential and dual role of this conserved Phe residue in MRP1 function appears exceptional. In this regard, it is worth noting that, other than Phe ${ }^{583}$, the sequence of MRP1-ECL5 has relatively limited homology with the comparable regions of CFTR and other ABCC homologs (Fig. 1B).
Through biochemical, modeling, and structural studies, CFTR-Phe ${ }^{337}$ has been shown by several groups to be accessible to the extracellular milieu and face in toward the aqueous pore of this chloride channel, which is consistent with its role in anion selectivity (Cheung and Akabas, 1997; Corradi et al., 2015; Linsdell, 2017; Liu et al., 2017). MRP1-Phe ${ }^{583}$ is similarly positioned according to both the TM287/288-based nucleotide-free inward-facing closed, and the outward-facing, nucleotide-bound Sav1866-based atomic homology models of human MRP1 (DeGorter et al., 2008), as well as in all three (apo-, LTC 4 -bound, and ATP-bound) cryoEM structures of bMrp1, published while this work was in progress (Johnson and Chen, 2017, 2018). Also similar to CFTR, the orientation of the MRP1-Phe ${ }^{583}$ side chain in the absence of nucleotide differs substantially from that when bound to nucleotide (Johnson and Chen, 2018), and in CFTR this is consistent with a gating function for this residue. However, rather than simply affecting the substrate selectivity of MRP1, the nucleotide interactions of MRP1-F583A were also substantially altered. These observations were unexpected because the exofacial location of $\mathrm{Ph}^{583}$ is far removed from the NBDs. Consequently, it is not immediately obvious how the loss of an aromatic side chain at position 583 can disrupt the ATPase activity of MRP1, particularly in the absence of substrate.

The two NBDs of ABC transporters dimerize to form two composite sites, each consisting of the Walker A and Walker $\mathrm{B}$ motifs from one NBD and the active transport signature motif of the other NBD (Dawson and Locher, 2007; Locher, 2016). In the case of MRP1 (and now a small subset of other ABC proteins including the TM287/288 heterodimer), it has been known for some time that these two sites are structurally and functionally nonequivalent (Cole et al., 1992; Gao et al., 2000; Hou et al., 2000, 2002; Nagata et al., 2000; Qin et al., 2008). One is now commonly referred to as the "consensus" (or "competent") site and the other as the "degenerate" site (Procko et al., 2006). The degenerate site binds ATP with high affinity, but has limited capacity to carry out hydrolysis, whereas the consensus site has a lower affinity for ATP, but has substantial hydrolytic activity. 
The F583A mutation did not affect the ability of MRP1 to bind ATP because the 8 -azido- $\left[{ }^{32} \mathrm{P}\right] \mathrm{ATP}$ photolabeling of the mutant was comparable to wild-type MRP1. In contrast, the orthovanadate-induced trapping of azido- $\left[{ }^{32} \mathrm{P}\right] \mathrm{ADP}$ (which reflects altered ATPase activity) was substantially greater than that of the wild-type transporter, even in the absence of substrate. Thus, the F583A mutation appears to disrupt MRP1 function through a long-range allosteric effect from ECL5 propagated to the cytoplasmic NBDs over a distance of some $75 \AA$ according to the bMrp1 cryoEM structures (Johnson and Chen, 2017). The increased level of $\left[{ }^{32} \mathrm{P}\right] \mathrm{ADP}$ trapping by the defective F583A mutant likely results from its diminished ability to release ADP after ATP hydrolysis, either through steric constraints imposed on the NBDs or through increased affinity for ADP. Irrespective of the mechanism, our observations indicate that the defective F583A mutant transporter is "locked" (trapped) in a posthydrolytic, low-affinity substrate binding state. This in turn points to a critical role for $\mathrm{Phe}^{583}$ (and more specifically, an aromatic side chain at this position) in the transition of MRP1 from its low-affinity substrate binding state to a high-affinity binding state during its transport cycle.

The arrangements of the TMs in the atomic homology models of human MRP1 bear a significant resemblance to the recently published cryoEM structures of bMrp1 (Johnson and Chen, 2017, 2018), and furthermore, as mentioned above, the models show how the binding of nucleotide changes the orientation of the $\mathrm{Phe}^{583}$ side chain. However, the two NBDs are much closer together (at least 3-fold) in the apo TM287/288-based model of human MRP1 than they are in either of the two nucleotide-free cryoEM structures of bMrp1 (Table 1). For the ATP-bound, hydrolysis-deficient bMrp1-E1454Q, the distance between the NBDs is substantially less and is comparable to that in the nucleotidebound $S$. aureus Sav1866-based homology model, but is only 1.5-fold less than the distances in the apo TM287/288based model (Table 1). Unfortunately, the current structures and models do not offer substantive mechanistic insights with respect to how the loss of the exofacial Phe $\mathrm{P}^{583}$ contributes to altered nucleotide interactions of MRP1.

To our knowledge, only one other MRP1 mutation outside its NBDs results in increased trapping of (post-ATP hydrolysis retention of) $\mathrm{ADP}$ as we observed here for F583A. Leu substitution of TM16-Glu ${ }^{1204}$ (which faces in toward the translocation pathway in all models and cryoEM structures) causes gross impairment of organic anion transport by MRP1 and a substantial increase in orthovanadate-induced trapping of azidoADP (Situ et al., 2004). However, in contrast to MRP1F583A, photolabeling of MRP1-E1204L by $\left[{ }^{3} \mathrm{H}\right] \mathrm{LTC}_{4}$ is comparable to wild-type MRP1. Thus, even though both the E1204L and F583A mutations disrupt the coupling of MRP1 ATPase activity to substrate transport, only the F583A mutant is defective in substrate binding.

It is conceivable that the replacement of MRP1-Phe ${ }^{583}$ with Ala causes a displacement or shift in the geometry of the COOH-proximal adjacent TM11, which, because it is linked to NBD1, disrupts the effective dimerization of NBD1 with NBD2. The pore-lining TM11 contains a high proportion of mutation-sensitive amino acids (Campbell et al., 2004; Haimeur et al., 2004; Koike et al., 2004; Zhang et al., 2004) (Fig. 1A), which is consistent with its critical role in substrate binding. In the case of the TM11-N590A mutant, a moderate loss of transport activity and $\mathrm{LTC}_{4}$ binding was also accompanied by a diminished ability to bind ATP (Zhang et al., 2004). However, unlike F583A, TM11-N590A showed no changes in orthovanadate-induced trapping of azidoADP. Thus, ECL5-Phe ${ }^{583}$ and the mutation-sensitive TM11 amino acids appear to play different roles in propagating signals between the MSDs and NBDs of MRP1.

In summary, our data indicate that not only is the outwardfacing $P h e^{583}$ important for maintaining the integrity of the substrate binding pockets of MRP1 but also for mediating long-range signaling that ensures functional interactions between NBD1 and NBD2. We propose that the loss of an aromatic side chain at position 583 biases MRP1 to favor a conformation with lower substrate affinity. This conformation also disrupts the functional cooperativity between the NBDs, such that posthydrolysis release of ADP is impaired although the binding of ATP remains intact. Further investigation is needed to understand precisely how long-range interactions between a single amino acid in an exofacial segment that spans the two 6-TM bundles of MRP1 and its two NBDs are transmitted. Additional biochemical studies guided by structures of wild-type and mutant MRP1 molecules in their various nucleotide- and substrate-bound forms, together with modeling and molecular dynamics simulations, should likely be helpful in this regard.

\section{Authorship Contributions}

Participated in research design: Weigl, Conseil, Rothnie, Arama, Tsfadia, and Cole.

Conducted experiments: Weigl, Conseil, Rothnie, and Arama.

Performed data analysis: Weigl, Conseil, Rothnie, Arama, Tsfadia, and Cole.

Wrote or contributed to the writing of the manuscript: Weigl, Conseil, Rothnie, Arama, Tsfadia, and Cole.

\section{References}

Amram S, Ganoth A, Tichon O, Peer D, Nachliel E, Gutman M, and Tsfadia Y (2014) Structural characterization of the drug translocation path of MRP1/ABCC1. Isr J Chem 54:1382-1393.

Borst P, Evers R, Kool M, and Wijnholds J (2000) A family of drug transporters: the multidrug resistance-associated proteins. J Natl Cancer Inst 92:1295-1302.

Campbell JD, Koike K, Moreau C, Sansom MSP, Deeley RG, and Cole SPC (2004) Molecular modeling correctly predicts the functional importance of $\mathrm{Phe}^{594}$ in transmembrane helix 11 of the multidrug resistance protein, MRP1 (ABCC1). $J$ Biol Chem 279:463-468.

Chen VB, Arendall WB, III, Headd JJ, Keedy DA, Immormino RM, Kapral GJ, Murray LW, Richardson JS, and Richardson DC (2010) MolProbity: all-atom structure validation for macromolecular crystallography. Acta Crystallogr D Biol Crystallogr 66:12-21.

Cheung M and Akabas MH (1997) Locating the anion-selectivity filter of the cystic fibrosis transmembrane conductance regulator (CFTR) chloride channel. J Gen Physiol 109:289-299.

Cole SPC (2014) Multidrug resistance protein 1 (MRP1, ABCC1), a "multitasking" ATP-binding cassette (ABC) transporter. J Biol Chem 289:30880-30888.

Cole SPC, Bhardwaj G, Gerlach JH, Mackie JE, Grant CE, Almquist KC, Stewart AJ, Kurz EU, Duncan AMV, and Deeley RG (1992) Overexpression of a transporter gene in a multidrug-resistant human lung cancer cell line. Science 258:1650-1654. Cole SPC, Sparks KE, Fraser K, Loe DW, Grant CE, Wilson GM, and Deeley RG (1994) Pharmacological characterization of multidrug resistant MRP-transfected human tumor cells. Cancer Res 54:5902-5910.

Conseil G, Rothnie AJ, Deeley RG, and Cole SPC (2009) Multiple roles of charged amino acids in cytoplasmic loop 7 for expression and function of the multidrug and organic anion transporter MRP1 (ABCC1). Mol Pharmacol 75:397-406.

Corradi V, Vergani P, and Tieleman DP (2015) Cystic fibrosis transmembrane conductance regulator (CFTR): closed and open state channel models. J Biol Chem 290:22891-22906.

Daoud R, Julien M, Gros P, and Georges E (2001) Major photoaffinity drug binding sites in multidrug resistance protein 1 (MRP1) are within transmembrane domains 10-11 and 16-17. J Biol Chem 276:12324-12330.

Dawson RJ and Locher KP (2006) Structure of a bacterial multidrug ABC transporter. Nature 443:180-185.

Dawson RJP and Locher KP (2007) Structure of the multidrug ABC transporter Sav1866 from Staphylococcus aureus in complex with AMP-PNP. FEBS Lett 581: 935-938. 
DeGorter MK, Conseil G, Deeley RG, Campbell RL, and Cole SPC (2008) Molecular modeling of the human multidrug resistance protein 1 (MRP1/ABCC1). Biochem Biophys Res Commun 365:29-34.

Gao M, Cui HR, Loe DW, Grant CE, Almquist KC, Cole SPC, and Deeley RG (2000) Comparison of the functional characteristics of the nucleotide binding domains of multidrug resistance protein $1 . J$ Biol Chem 275:13098-13108.

Haimeur A, Conseil G, Deeley RG, and Cole SPC (2004) Mutations of charged amino acids in or near the transmembrane helices of the second membrane spanning domain differentially affect the substrate specificity and transport activity of the multidrug resistance protein MRP1 (ABCC1). Mol Pharmacol 65:1375-1385.

Hegyi P, Wilschanski M, Muallem S, Lukacs GL, Sahin-Tóth M, Uc A, Gray MA, Rakonczay Z, Jr, and Maléth J (2016) CFTR: a new horizon in the pathomechanism and treatment of pancreatitis. Rev Physiol Biochem Pharmacol 170: 37-66.

Hipfner DR, Almquist KC, Stride BD, Deeley RG, and Cole SPC (1996) Location of a protease-hypersensitive region in the multidrug resistance protein (MRP) by mapping of the epitope of MRP-specific monoclonal antibody QCRL-1. Cancer Res 56:3307-3314

Hohl M, Hürlimann LM, Böhm S, Schöppe J, Grütter MG, Bordignon E, and Seeger MA (2014) Structural basis for allosteric cross-talk between the asymmetric nucleotide binding sites of a heterodimeric ABC exporter. Proc Natl Acad Sci USA 111:11025-11030.

Hou Y, Cui L, Riordan JR, and Chang X (2000) Allosteric interactions between the two non-equivalent nucleotide binding domains of multidrug resistance protein MRP1. J Biol Chem 275:20280-20287.

Hou YX, Cui L, Riordan JR, and Chang XB (2002) ATP binding to the first nucleotidebinding domain of multidrug resistance protein MRP1 increases binding and hydrolysis of ATP and trapping of ADP at the second domain. J Biol Chem 277: $5110-5119$.

Jansen RS, Küçükosmanoglu A, de Haas M, Sapthu S, Otero JA, Hegman IE, Bergen AA, Gorgels TG, Borst P, and van de Wetering K (2013) ABCC6 prevents ectopic mineralization seen in pseudoxanthoma elasticum by inducing cellular nucleotide release. Proc Natl Acad Sci USA 110:20206-20211.

Johnson ZL and Chen J (2017) Structural basis of substrate recognition by the multidrug resistance protein MRP1. Cell 168:1075-1085.e9.

Johnson ZL and Chen J (2018) ATP binding enables substrate release from multidrug resistance protein 1. Cell 172:81-89.e10.

Karwatsky J, Daoud R, Cai J, Gros P, and Georges E (2003) Binding of a photoaffinity analogue of glutathione to MRP1 (ABCC1) within two cytoplasmic regions (L0 and L1) as well as transmembrane domains $10-11$ and 16-17. Biochemistry 42 $3286-3294$

Keppler D (2011) Multidrug resistance proteins (MRPs, ABCCs): importance for pathophysiology and drug therapy. Handb Exp Pharmacol 201:299-323.

Koike K, Conseil G, Leslie EM, Deeley RG, and Cole SPC (2004) Identification of proline residues in the core cytoplasmic and transmembrane regions of multidrug resistance protein 1 (MRP1/ABCC1) important for transport function, substrate specificity, and nucleotide interactions. J Biol Chem 279:12325-12336.

Koike K, Oleschuk C.J, Haimeur A, Olsen SL, Deeley RG, and Cole SPC (2002) Multiple membrane-associated tryptophan residues contribute to the transport activity and substrate specificity of the human multidrug resistance protein MRP1. J Biol Chem 277:49495-49503.

Laskowski RA, MacArthur MW, Moss DS, and Thornton JM (1993) PROCHECK - a program to check the stereochemical quality of protein structures. J Appl Cryst $\mathbf{2 6}$ $283-291$

Le Saux O, Beck K, Sachsinger C, Silvestri C, Treiber C, Göring HHH, Johnson EW, De Paepe A, Pope FM, Pasquali-Ronchetti I, et al. (2001) A spectrum of ABCC6 mutations is responsible for pseudoxanthoma elasticum. Am J Hum Genet 69. $749-764$.

Leslie EM (2012) Arsenic-glutathione conjugate transport by the human multidrug resistance proteins (MRPs/ABCCs). J Inorg Biochem 108:141-149.

Leslie EM, Ito K, Upadhyaya P, Hecht SS, Deeley RG, and Cole SPC (2001) Transport of the $\beta$-O-glucuronide conjugate of the tobacco-specific carcinogen 4-(methylnitrosamino)-1-(3-pyridyl)-1-butanol (NNAL) by the multidrug resistance protein 1 (MRP1). Requirement for glutathione or a non-sulfur-containing analog. $J$ Biol Chem 276:27846-27854.

Lindorff-Larsen K, Piana S, Palmo K, Maragakis P, Klepeis JL, Dror RO, and Shaw DE (2010) Improved side-chain torsion potentials for the Amber ff99SB protein force field. Proteins 78:1950-1958.

Linsdell P (2017) Architecture and functional properties of the CFTR channel pore. Cell Mol Life Sci 74:67-83.

Linsdell P, Evagelidis A, and Hanrahan JW (2000) Molecular determinants of anion selectivity in the cystic fibrosis transmembrane conductance regulator chloride channel pore. Biophys J 78:2973-2982.

Liu F, Zhang Z, Csanády L, Gadsby DC, and Chen J (2017) Molecular structure of the human CFTR ion channel. Cell 169:85-95.e8.

Locher KP (2016) Mechanistic diversity in ATP-binding cassette (ABC) transporters Nat Struct Mol Biol 23:487-493.

Loe DW, Almquist KC, Deeley RG, and Cole SPC (1996) Multidrug resistance protein (MRP)-mediated transport of leukotriene $\mathrm{C} 4$ and chemotherapeutic agents in membrane vesicles. Demonstration of glutathione-dependent vincristine transport. J Biol Chem 271:9675-9682.

Loe DW, Deeley RG, and Cole SPC (1998) Characterization of vincristine transport by the M(r) 190,000 multidrug resistance protein (MRP): evidence for cotransport with reduced glutathione. Cancer Res 58:5130-5136.

Maeno K, Nakajima A, Conseil G, Rothnie A, Deeley RG, and Cole SPC (2009) Molecular basis for reduced estrone sulfate transport and altered modulator sensitivity of transmembrane helix (TM) 6 and TM17 mutants of multidrug resistance protein 1 (ABCC1). Drug Metab Dispos 37:1411-1420.

Nagata K, Nishitani M, Matsuo M, Kioka N, Amachi T, and Ueda K (2000) Nonequivalent nucleotide trapping in the two nucleotide binding folds of the human multidrug resistance protein MRP1. J Biol Chem 275:17626-17630.

Peklak-Scott C, Townsend AJ, and Morrow CS (2005) Dynamics of glutathione conjugation and conjugate efflux in detoxification of the carcinogen, 4-nitroquinoline 1-oxide: contributions of glutathione, glutathione S-transferase, and MRP1. Biochemistry 44:4426-4433.

Petrova SS and Solov'ev AD (1997) The origin of the method of steepest descent. Hist Math 24:361-375.

Procko E, Ferrin-O'Connell I, Ng SL, and Gaudet R (2006) Distinct structural and functional properties of the ATPase sites in an asymmetric ABC transporter. Mol Cell 24:51-62.

Qian YM, Song WC, Cui H, Cole SPC, and Deeley RG (2001) Glutathione stimulates sulfated estrogen transport by multidrug resistance protein 1 . J Biol Chem 276: 6404-6411.

Qin L, Zheng J, Grant CE, Jia Z, Cole SPC, and Deeley RG (2008) Residues responsible for the asymmetric function of the nucleotide binding domains of multidrug resistance protein 1. Biochemistry 47:13952-13965.

Rappa G, Lorico A, Flavell RA, and Sartorelli AC (1997) Evidence that the multidrug resistance protein (MRP) functions as a co-transporter of glutathione and natura product toxins. Cancer Res 57:5232-5237.

Riordan JR (2008) CFTR function and prospects for therapy. Annu Rev Biochem 77: $701-726$.

Rosenberg MF, Oleschuk CJ, Wu P, Mao Q, Deeley RG, Cole SPC, and Ford RC (2010) Structure of a human multidrug transporter in an inward-facing conformation. J Struct Biol 170:540-547.

Rothnie A, Callaghan R, Deeley RG, and Cole SPC (2006) Role of GSH in estrone sulfate binding and translocation by the multidrug resistance protein 1 (MRP1/ABCC1). J Biol Chem 281:13906-13914.

Situ D, Haimeur A, Conseil G, Sparks KE, Zhang D, Deeley RG, and Cole SPC (2004) Mutational analysis of ionizable residues proximal to the cytoplasmic interface of membrane spanning domain 3 of the multidrug resistance protein, MRP1 (ABCC1): glutamate 1204 is important for both the expression and catalytic activity of the transporter. J Biol Chem 279:38871-38880.

Slot AJ, Molinski SV, and Cole SPC (2011) Mammalian multidrug-resistance proteins (MRPs). Essays Biochem 50:179-207.

Tabas LB and Dantzig AH (2002) A high-throughput assay for measurement of multidrug resistance protein-mediated transport of leukotriene $\mathrm{C}_{4}$ into membrane vesicles. Anal Biochem 310:61-66.

Uitto J, Jiang Q, Váradi A, Bercovitch LG, and Terry SF (2014) Pseudoxanthoma elasticum: diagnostic features, classification, and treatment options. Expert Opin Orphan Drugs 2:567-577.

Urbatsch IL, Sankaran B, Weber J, and Senior AE (1995) P-glycoprotein is stably inhibited by vanadate-induced trapping of nucleotide at a single catalytic site. $J$ Biol Chem 270:19383-19390.

Van Der Spoel D, Lindahl E, Hess B, Groenhof G, Mark AE, and Berendsen HJC (2005) GROMACS: fast, flexible, and free. J Comput Chem 26:1701-1718.

Wei S, Roessler BC, Icyuz M, Chauvet S, Tao B, Hartman JL, IV, and Kirk KL (2016 Long-range coupling between the extracellular gates and the intracellular ATP binding domains of multidrug resistance protein pumps and cystic fibrosis transmembrane conductance regulator channels. FASEB $J$ 30:1247-1262.

Zaman GJ, Lankelma J, van Tellingen O, Beijnen J, Dekker H, Paulusma C, Oude Elferink RP, Baas F, and Borst P (1995) Role of glutathione in the export of compounds from cells by the multidrug-resistance-associated protein. Proc Natl Acad Sci USA 92:7690-7694.

Zhang DW, Nunoya K, Vasa M, Gu HM, Cole SPC, and Deeley RG (2006) Mutational analysis of polar amino acid residues within predicted transmembrane helices 10 and 16 of multidrug resistance protein 1 (ABCC1): effect on substrate specificity. Drug Metab Dispos 34:539-546.

Zhang DW, Nunoya K, Vasa M, Gu HM, Theis A, Cole SPC, and Deeley RG (2004) Transmembrane helix 11 of multidrug resistance protein 1 (MRP1/ABCC1): identification of polar amino acids important for substrate specificity and binding of ATP at nucleotide binding domain 1. Biochemistry 43:9413-9425.

Address correspondence to: Dr. Susan P. C. Cole, Division of Cancer Biology and Genetics, Queen's University Cancer Research Institute, Room 312, 10 Stuart Street, Kingston, ON K7L 3N6, Canada. E-mail: spc.cole@ queensu.ca 